Article

\title{
Multipath Data Storage by Third-Order Nonlinear Optical Properties in Zinc Oxide Nanostructures
}

\author{
Federico Ortíz-Trejo ${ }^{1}$, Martín Trejo-Valdez ${ }^{1}$, Juan Pablo Campos-López ${ }^{2}$, \\ Joel Humberto Castro-Chacón ${ }^{3}$ and Carlos Torres-Torres ${ }^{4, *}$ (1) \\ 1 Escuela Superior de Ingeniería Química e Industrias Extractivas, Instituto Politécnico Nacional, \\ Ciudad de México 07738, Mexico; fortiz34@yahoo.com (F.O.-T.); martin.trejo@laposte.net (M.T.-V.) \\ 2 División de Ingeniería en Mecatrónica, Universidad Politécnica del Valle de México, Tultitlán, \\ Estado de México 54910, Mexico; campos_juan_pablo@yahoo.com \\ 3 CONACYT-Instituto de Astronomía Universidad Nacional Autónoma de México, Ensenada 22860, Mexico; \\ joelhcch@gmail.com \\ 4 Sección de Estudios de Posgrado e Investigación, Escuela Superior de Ingeniería Mecánica y Eléctrica \\ Unidad Zacatenco, Instituto Politécnico Nacional, Ciudad de México 07738, Mexico \\ * Correspondence: ctorrest@ipn.mx or crstorres@yahoo.com.mx
}

Received: 20 May 2020; Accepted: 15 June 2020; Published: 17 August 2020

check for updates

\begin{abstract}
Simultaneous activation of multiphotonic absorption and nonlinear optical refraction were employed for developing a multipath load balancing technique assisted by light and zinc oxide nanostructures. Data storage as the real and imaginary part of the third-order nonlinear optical behavior of different regions in bidimensional systems was proposed. By recording the third-order nonlinear optical response exhibited by the nanomaterials, a straightforward XOR encryption system controlled by laser pulses was developed. Identification of nonlinear optical signals in propagation through the samples was monitored by a standard optical Kerr gate configuration. Vectorial two-wave mixing experiments were carried out for measuring the third-order optical nonlinearities of the samples explored by nanosecond pulses at $532 \mathrm{~nm}$ wavelength. A spray pyrolysis processing route was employed for the preparation of the samples in thin film form. Third-order nonlinear optical characteristics were analyzed taking into account the modification in the $\mathrm{ZnO}$-based nanocomposites by single-pulsed irradiation close to the ablation threshold effect. The combination of nonlinear optics and nanostructured films can be considered for developing ultrafast smart objects with immediate applications for signal processing functions driven by multiphotonic phenomena.
\end{abstract}

Keywords: nonlinear optics; two-wave mixing; encryption; $\mathrm{ZnO}$; nanostructures; thin solid films

\section{Introduction}

All-optical interconnection among objects corresponds to a fascinating area of data research considering the imminent evolution of the speed in random uniform functions. Ubiquitous sensing technologies seem to be potentially enabled by ultrafast communications that may be associated with nonlinear optical phenomena. The challenge of transferring information by nonlinear optical signals in general involves high irradiance laser systems interacting with advanced materials [1]. In this respect, light controlled by light must be ascribed to a powerful enhancement in electromagnetic effects with the assistance of multiphotonic processes [2]. Third-order nonlinear optical properties present a strong dependence on morphology and structure of nanomaterials [3]. Highly transparent nanoparticles embedded in diverse dielectrics have been proposed as good candidates for designing nonlinear optical modulating systems [4]. However, zinc oxide materials represent an excellent alternative in regards to their optical characteristics, electronic properties and high transparency in nanostructured form [5]. 
$\mathrm{ZnO}$ nanostructures have been useful as a powerful tool in electronics [6], nanomedicine [7], optical communications [8], bioinspired systems [9] and actuators [10]. Their ultraviolet band gap may be particularly useful for developing quantum and single-photon devices [11].

Thin solid films build up by $\mathrm{ZnO}$ doped with metals are considered an important option to synthetize nanostructures with controlled size and shape. Particularly, monometallic nanoparticles correspond to a Localized Surface Plasmon Resonance phenomenon that derives in a sharp selective optical response. However, it is worth mentioning that metals in nanocluster form may correspond to proto-plasmonic effects not observed in the optical absorption spectrum but with implications for the enhancement of third-order optical nonlinearities [12].

Nanoscale optical effects are responsible for an outstanding topic in sensing, signal processing and decision making [13]. In addition to the parameters of irradiance and wavelength, the polarization of light also represents a clear influence on the coupling and modulation of nanoscopic phenomena [14].

It can be considered that the optical Kerr effect and the nonlinear optical absorption should be strong to be useful in many practical applications. The participation of multicomponent nanostructures can be considered a solution for tailoring multifunctional systems able to perform synergistic effects and resonance conditions in two or more wavelengths of the electromagnetic spectrum [15].

In order to improve the third-order nonlinear optical properties exhibited by $\mathrm{ZnO}$ nanostructures, different volume fractions of $\mathrm{ZnO}$ with different elements have been proposed [16]. A fundamental key for enhancing the third-order optical effects is the relation between area and volume in hybrid nanocomposites. Remarkably, the preparation method involved in the synthesis of metal doped $\mathrm{ZnO}$ seems to determine specific characteristics exhibited by the samples in thin film form [17].

Diverse studies have reported the potential of nonlinear optical properties exhibited by $\mathrm{ZnO}$ nanocomposites in all-optical switching [18] and imaging [19]. It has been pointed out that the integration of nonlinear optics and quantum confinement effects may drive a groundbreaking advance in favor to the progress for encoding information in light [20]. The influence of nonlinear optical processes for encrypting operations has been contemplated [21]. Basic computing functions based on third-order optical nonlinearities exhibited by different $\mathrm{ZnO}$ nanostructures have been analyzed and nanophotonic circuits have been envisioned [22]. With all these motivations, this work has been focused to further investigate the real and imaginary parts of the third-order nonlinear optical properties exhibited by $\mathrm{ZnO}$ nanostructures. A multipath load balancing technique was proposed by using nonlinear optical effects induced by nanosecond pulses in a two-wave mixing configuration. An optical logic gate was employed for identifying optical nonlinearities in the thin solid films that were studied. The modification in multiphotonic interactions related to the optical Kerr effect and two-photon absorption exhibited by the 2D nanostructures were analyzed. These findings could be a base for future research related to the development of all-optical interconnection functions. This article has analyzed the potential of engineering optical nonlinearities for instrumentation and processing signals in multipath configurations.

\section{Materials and Methods}

\subsection{Sample Synthesis}

The solid film $\mathrm{ZnO}$ samples deposited on silica substrates were prepared by a starting solution with zinc (II) acetate $\left[\mathrm{Zn}\left(\mathrm{O}_{2} \mathrm{CCH}_{3}\right)_{2}\right]$ exhibiting $0.2 \mathrm{M}$ in a mix of deionized water, methanol $\left[\mathrm{CH}_{3} \mathrm{OH}\right]$ and acetic acid $\left[\mathrm{CH}_{3} \mathrm{CO}_{2} \mathrm{H}\right]$ in a proportion of 1:8:1 volume relation, respectively. In parallel, silver nitrate [Ag $\left.\left(\mathrm{NO}_{3}\right)\right]$ with $0.25 \mathrm{M}$ was dissolved in a mix of deionized water and acetic acid $\left[\mathrm{CH}_{3} \mathrm{CO}_{2} \mathrm{H}\right]$ (1:1 volume relation). We employed a constant $[\mathrm{Ag}] /[\mathrm{Zn}]$ atomic percent ratio of 0.1 at. $\%$ in the preparation process in order to modulate the $\mathrm{NO}_{3}$ action. A flow rate of $0.5 \mathrm{~L} / \mathrm{min}$ of $\mathrm{N}_{2}$ was used as the solution carrier and director gas in the deposition. A piezoelectric transducer with $1.2 \mathrm{MHz}$ and $120 \mathrm{~W}$ of ultrasonic power in the synthesis process was employed for the preparation of the samples [23]. 
The silica substrates have $5 \mathrm{~cm}^{2}$ of area and the thickness of the substrate was $1 \mathrm{~mm}$. During the processing route, the substrate temperature was $400^{\circ} \mathrm{C}$ and, after deposition, the samples were annealed in the same temperature for $4 \mathrm{~h}$ in order to promote crystallization. Thin solid films were obtained and, by using ellipsometry evaluations, samples with $500 \mathrm{~nm}$ of thickness were selected for the study of the optical properties. Morphology observations were carried out by a scanning electronic microscopy (SEM) studies in a FEI Quanta 3D FEG microscope system in STEM mode (scanning transmission electronic microscopy). X-ray diffraction (XRD) measurements were undertaken by a Phillips X'pert diffractometer in order to reveal the crystalline structure of the $\mathrm{ZnO}$ thin films. The parameters of operation of the XRD system correspond to $40 \mathrm{kV}$ and $30 \mathrm{~mA}$ using $\mathrm{Cu} \mathrm{K} \alpha$ radiation.

Single pulses below the ablation threshold of the nanostructures were propagated through different regions of the film in order to promote a potential laser annealing process. Single-shots with maximum fluence of energy per pulse $3.5 \mathrm{~J} / \mathrm{cm}^{2}$ at $532 \mathrm{~nm}$ and 4 nanosecond of pulse duration were employed in a focused beam waist of $1 \mathrm{~mm}$ of diameter. More than a hundred regions were explored. The ablation threshold of the sample was measured to be $4 \mathrm{~J} / \mathrm{cm}^{2}$ in the same laser conditions of wavelength and pulse duration. The modification of the nonlinear optical properties exhibited by as-prepared samples and the optically irradiated samples were systematically compared.

\subsection{Nanosecond Third-Order Nonlinear Optical Studies}

Multiphotonic phenomena associated with third-order nonlinear optical properties were conducted in the samples by using the linearly polarized second harmonic of a Nd-YAG laser system (Continuum Model SL II). A vectorial two-wave mixing experiment and a single-beam transmittance setup were employed with a pulsed energy of $110 \mathrm{~mJ}$ maximum, an optical wavelength at $532 \mathrm{~nm}$ and single-shot mode of pulses with 4 nanoseconds of FWHM duration. A beam waist of $1 \mathrm{~mm}$ of diameter was focused in the sample with a relation of optical irradiance 1:1 between the incident beams. The probe and pump beams were making a geometric angle of approximately $10^{\circ}$ at the surface of the film. The polarization of the transmitted probe beam was analyzed by a calcite polarizer located behind the sample. PIN photodetectors were employed for acquiring the nonlinear transmittance and the orthogonal component of the polarization of the transmitted probe beam. The vectorial nature of this experiment was evaluated by the assistance of a half-wave plate able to rotate the polarization of the pump beam during the measurements while the polarization of the probe beam was fixed. The calibration of the two-wave mixing experiment was carried out by a $\mathrm{CS}_{2}$ liquid solution deposited in a $1 \mathrm{~mm}$ length quartz cuvette. The error bar in our measurements was approximately $\pm 10 \%$.

The wave equation was employed for the mathematical representation of the amplitudes of the electric fields in propagation through the samples [24]:

$$
\nabla^{2} E_{ \pm}=-\frac{n_{ \pm}^{2} \omega^{2}}{c^{2}} E_{ \pm}
$$

Here the optical frequency is $\omega$ and $c$ is the speed of the light. $E_{+}$and $E_{-}$correspond to the circular components of the right and left polarized electric fields, respectively. The refractive index, $n_{ \pm}$, can be approximated by [24]

$$
n_{ \pm}^{2}=n_{0}^{2}+4 \pi\left(A\left|E_{ \pm}\right|^{2}+(A+B)\left|E_{\mp}\right|^{2}\right)
$$

where $n_{0}$ is the weak-field refractive index, the refractive index dependent on optical irradiance in optically isotropic materials involves independent components of the third-order susceptibility tensor, $\chi^{(3)}$, described by $A=\chi_{1122}^{(3)}$ and $B=\chi_{1212}^{(3)}$. We described $\chi^{(3)}$ by [24]

$$
\chi_{1111}^{(3)}=\frac{n_{0} c}{7.91 \times 10^{2}} n_{2}+i \frac{n_{0}^{2} c \lambda}{\pi^{2}} \beta
$$


In addition, the transmitted optical irradiance $I(L)$ is [25]

$$
I(L)=\frac{I_{o} \exp \left(-\alpha_{0} L\right)}{1+\beta I_{o} L_{e f f}},
$$

Considering initial incident irradiance $I_{0}$, and $\alpha_{0}$ as the optical absorption coefficient at low irradiance, then the effective length $L_{\text {eff }}$ is

$$
L_{e f f}=\frac{\left(1-\exp \left(-\alpha_{0} L\right)\right)}{\alpha_{0}}
$$

\section{Results}

\subsection{Nanosecond Third-Order Nonlinear Optical Studies}

The nonlinear optical absorption behavior of a material can be observed through the observation of the optical transmittance as a function of the optical intensity irradiating a sample. We determined the ablation threshold of the sample by a single pulse with fluence $4 \mathrm{~J} / \mathrm{cm}^{2}$. From Figure 1 can be noticed that a monotonic flat trace demonstrates the fingerprint of a two-photon absorption exhibited by the samples. The fitting by Equation (4) was made considering a nonlinear optical absorption coefficient $\beta=4.4 \times 10^{-9} \mathrm{~m} / \mathrm{W}$. We noticed that the irradiation of a single pulse $10 \%$ below the ablation threshold of the sample gives origin to a fitted $\beta=11.9 \times 10^{-9} \mathrm{~m} / \mathrm{W}$ as can be seen in Figure 1, but no changes in $L_{\text {eff }}$ were observed.

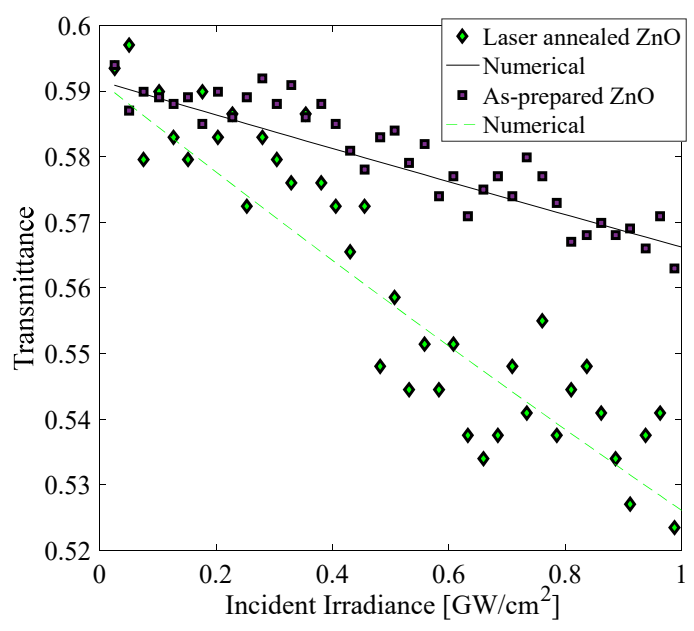

Figure 1. Evolution of the optical transmittance as a function of the incident irradiance in the samples studied by nanosecond pulses at $532 \mathrm{~nm}$ in a single-beam configuration.

\subsection{Non-Degenerated Two-Wave Mixing at 532 nm by Nanosecond Pulses}

The Kerr transmittance obtained by a two-wave mixing allowed us to monitor the evolution of the polarization of the probe beam under the influence of the probe beam in the experiment. In Figure 2 is plotted the modulation of the transmitted probe beam as a function of the geometric angle between the planes of polarization of the incident beams. The inhibition of the Kerr signal for parallel and orthogonal polarizations $\left(0^{\circ}\right.$ and $90^{\circ}$ in the axis of the abscissa in the plot) pointed out a thermal effect as the main physical mechanism responsible for the third-order optical nonlinearity.

The asymmetric response in the probe transmitted experiment illustrated in Figure 2 can be correlated with a homogeneous distribution of the nanostructures in the sample. The fitting in the magnitude of third-order nonlinear optical susceptibility for the samples results in a maximum $\left|\chi_{e f f}^{(3)}\right|=2.55 \times 10^{-9}$ esu. However, during the identification of the ablation threshold of the sample, 
we noticed the potential modulation of the third-order nonlinear optical response by an irradiation process below the photodamage.

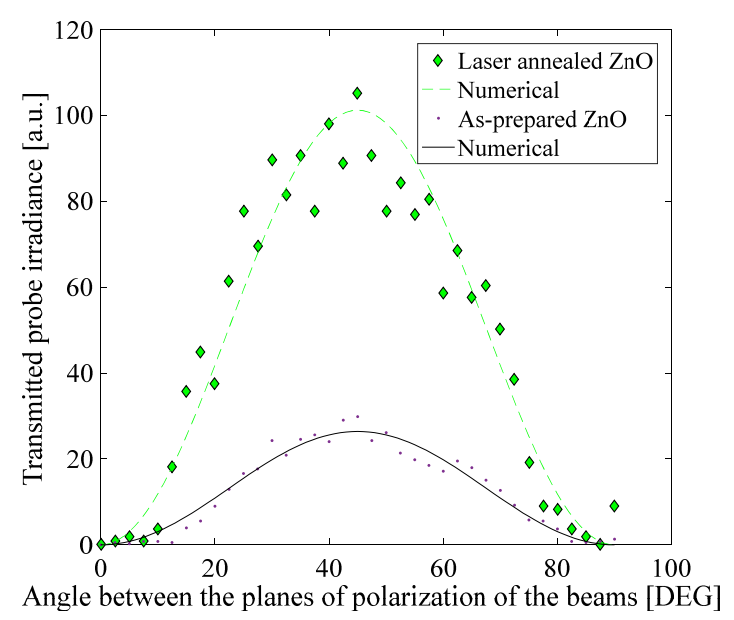

Figure 2. Nanosecond probe transmittance vs. angle of polarization of the interacting beams in the thin films studied.

We irradiated different regions of the samples with single-shots with maximum energy per pulse $3.5 \mathrm{~J} / \mathrm{cm}^{2}$. Two-wave mixing experiments and single-beam measurements in the different irradiated regions confirmed anisotropic absorptive and refractive nonlinearities, but constant optical absorption evaluated by spectroscopy at $532 \mathrm{~nm}$ wavelength was guaranteed. Then we estimated $n_{2}$ and $\beta$ using Equation (3) and the results are shown in Table 1.

Table 1. Optical parameters.

\begin{tabular}{ccccccc}
\hline Sample & $\begin{array}{c}\boldsymbol{\alpha}_{0} \\
{\left[\mathrm{~cm}^{-1}\right]}\end{array}$ & $\begin{array}{c}\text { Bandgap } \\
{[\mathrm{eV}]}\end{array}$ & $n_{0}$ & $\begin{array}{c}n_{2} \\
{\left[\mathbf{m}^{2} / \mathbf{W}\right]}\end{array}$ & $\begin{array}{c}\beta \\
{[\mathrm{m} / \mathbf{W}]}\end{array}$ & $\begin{array}{c}\mid \chi_{\text {eff }}^{(3)}[ \\
{[\mathbf{e s u}]}\end{array}$ \\
\hline As-prepared nanostructures & $1.0541 \times 10^{4}$ & 3.1813 & 1.95 & $1.7 \times 10^{-15}$ & $4.4 \times 10^{-9}$ & $2.54 \times 10^{-9}$ \\
\hline Laser annealed nanostructures & $1.0735 \times 10^{4}$ & 3.2059 & 1.95 & $5.9 \times 10^{-15}$ & $11.9 \times 10^{-9}$ & $8.80 \times 10^{-9}$ \\
\hline
\end{tabular}

\subsection{Morphology and Spectroscopy of the Samples}

The modulation in the third-order nonlinear optical effects in the laser annealed $\mathrm{ZnO}$ nanostructures was obtained with no observation of changes in optical absorption nor in their morphology. Microscopy evidences for the nanostructured morphology exhibited by the $\mathrm{ZnO}$ thin solid film samples are shown in Figure 3. Statistical measurements indicate that the grain size corresponds to particles with a distribution from 100 to $500 \mathrm{~nm}$. The pyramidal shape of the columnar nanostructures seems to be a signature for the defects and dislocation in these nanostructures [23].

In Figure 4 is shown a representative XRD pattern measured in the $\mathrm{ZnO}$ samples studied in this work. No differences were identified in the as-prepared sample in comparison to the laser annealed sample. The $\mathrm{ZnO}$ nanostructures correspond to an indexation related to zinc oxide hexagonal (wurzite) phase consistent with the standard values for ZnO bulk (JCPDS Cardno. 36-1451). No evidence of presence of $\mathrm{Ag}$ in the films was detected by exhaustive XRD exploration measurements.

High transparency in the films was revealed by spectroscopy observations. As it could be expected, from Figure 5 can be seen a monotonic increase in the absorption in the UV region of the electromagnetic spectra. However, only a small change in the optical absorption for the comparative samples at the $532 \mathrm{~nm}$ wavelength can be clearly visualized. 


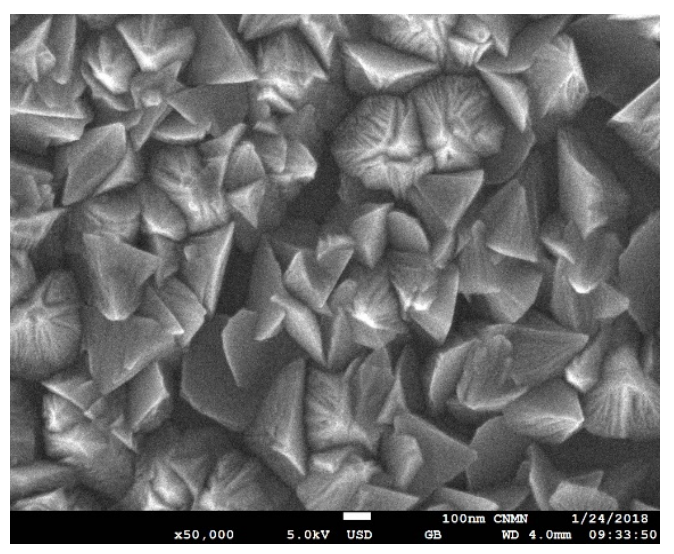

Figure 3. Typical SEM micrograph of the $\mathrm{ZnO}$ thin solid film.

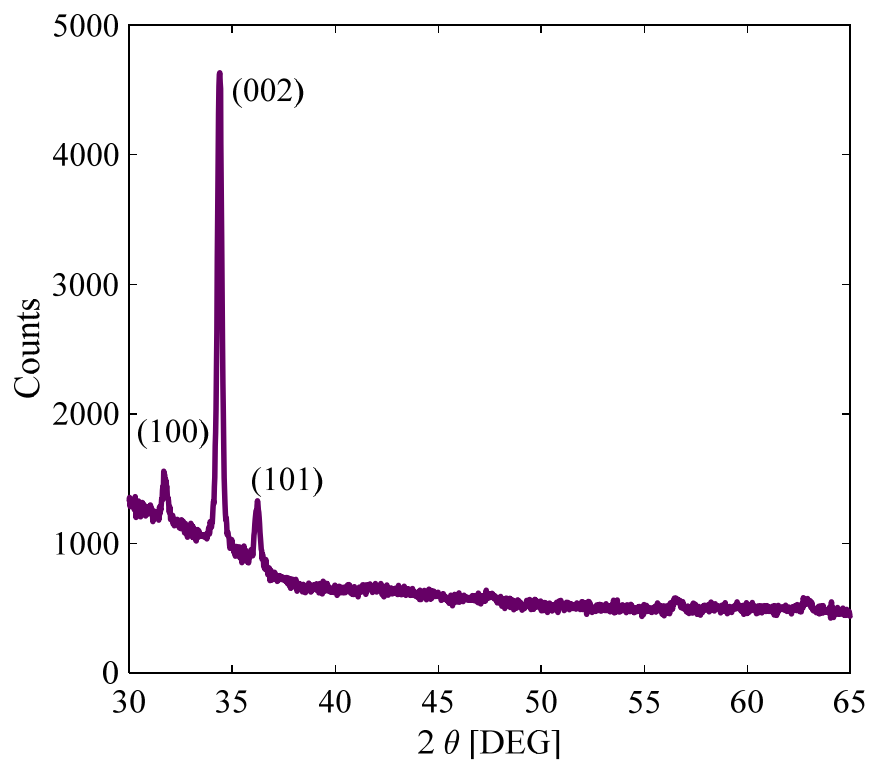

Figure 4. XRD pattern showing the principal planes measured in the studied $\mathrm{ZnO}$ sample.

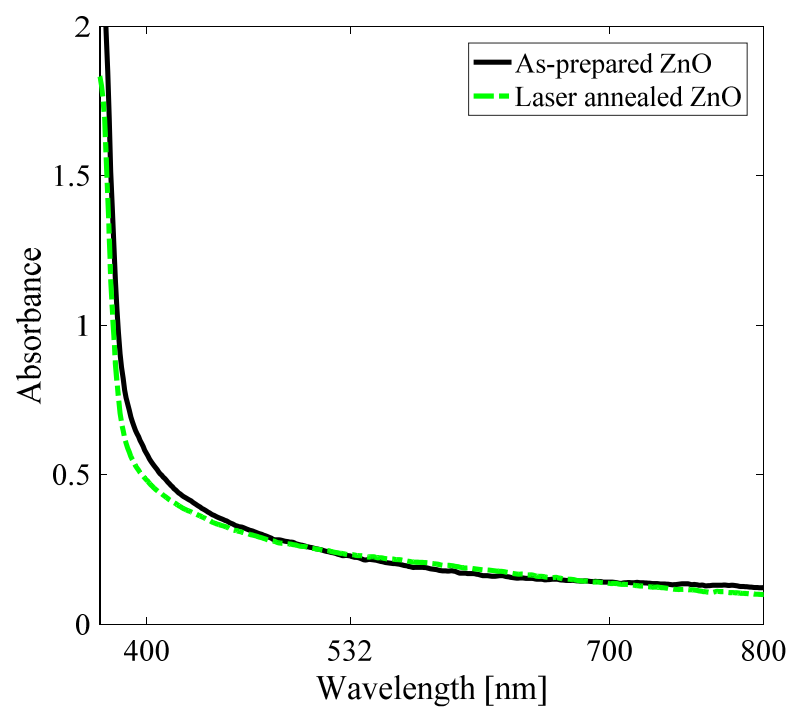

Figure 5. Representative UV-vis absorbance spectra of the $\mathrm{ZnO}$ samples. 


\subsection{Multipath Load Balancing Assisted by Nonlinear Optical Properties}

In order to explain the drastic increase in the nonlinear optical response derived by the laser annealing process in the sample, we identified that the modification in optical losses automatically changes the nonlinear response. The distribution of the nanostructures in the film is random but the collective third-order optical nonlinearities are dependent on radiative and non-radiative processes. In this regard, we clearly noticed changes in UV-vis spectra by laser annealing. Changes in morphology or structure in the film measured by TEM and XRD techniques were not observed. However, it can be expected that the removal of defects can be obtained by laser annealing regarding the observed modification in the multiphotonic transitions of the film. The third-order nonlinear optical response exhibited by a nonlinear system is dependent on atomic dipole moment. Formation and elimination of localized electronic states induced by thermodynamic passivation of $\mathrm{ZnO}$ surfaces has been reported [26]. Then magnetic techniques seem to be suitable for revealing with high sensitivity changes in electronic bonds by the incorporation of removal of defects.

We describe $\chi^{(3)}$, considering a nanosecond radiation with optical frequency $\omega$, and a detuning of frequency represented by $\Delta=\omega-\omega_{21}$, then [27]

$$
\chi^{(3)}=\frac{4 N m^{4}\left[T_{1} T_{2}^{2}\left(\Delta T_{2}-i\right)\right]}{3 \hbar^{3}\left[1+\Delta T_{2}^{2}\right]^{2}} .
$$

In Equation (6), $N$ describes two-level atoms per unit volume, $\hbar$ describes the Planck constant, $m$ is associated with the atomic dipole moment, $1 / T_{1}$ describes the population loss through radiative and non-radiative processes of the upper quantum level of excitation, and $1 / T_{2}$ describe the dynamics of a two-level system with a characteristic rate of polarization loss.

In our studied case, the laser annealing seems to derive in a modification in the multiphotonic transitions of the film. In the limit of large detunings, i.e., $\Delta T_{2} \gg 1$, it can be considered that [27]

$$
\operatorname{Re} \chi^{(3)} \approx \frac{4}{3} N m^{4}\left[\frac{1}{\hbar \Delta}\right]^{3} \frac{T_{1}}{T_{2}} .
$$

In addition, the same consideration can be obtained [28],

$$
\operatorname{Im} \chi^{(3)} \approx-\frac{4}{3} N m^{4}\left[\frac{1}{\hbar \Delta}\right]^{3} \frac{T_{1}}{\Delta T_{2}^{2}}
$$

Regarding that no modification in nanostructured morphology of the samples was obtained after the optical irradiation, the changes in both refractive and absorptive nonlinearities are not suitable to be described by $N$ or $m$ in this model. On the other hand, small modifications related to changes in $\Delta$ or in population losses can generate a strong enhancement in the third-order optical nonlinear properties. In a non-resonant regime, as is our case, the radiative spontaneous emission and the decay due to phonons could be promoted by inhibition of defects in the nanostructures, and these conditions must be more important in respect to the non-radiative spontaneous emission of the atoms. This consideration as a function on the modification of the loss factor $T_{1} / T_{2}$ is shown in Figure 6 .

We consider an XOR operation in order to identify binary data recorded in the sample. An exclusive-OR logic gate generates a logical zero if the operands are assumed to be identical; otherwise, a logical value equal to one is obtained. In this work, the operation principle underlying this function was proposed to be by two different paths; these channels correspond to the separated evaluation of the real and imaginary part of $\chi^{(3)}$, measured by a standard two-wave mixing. 


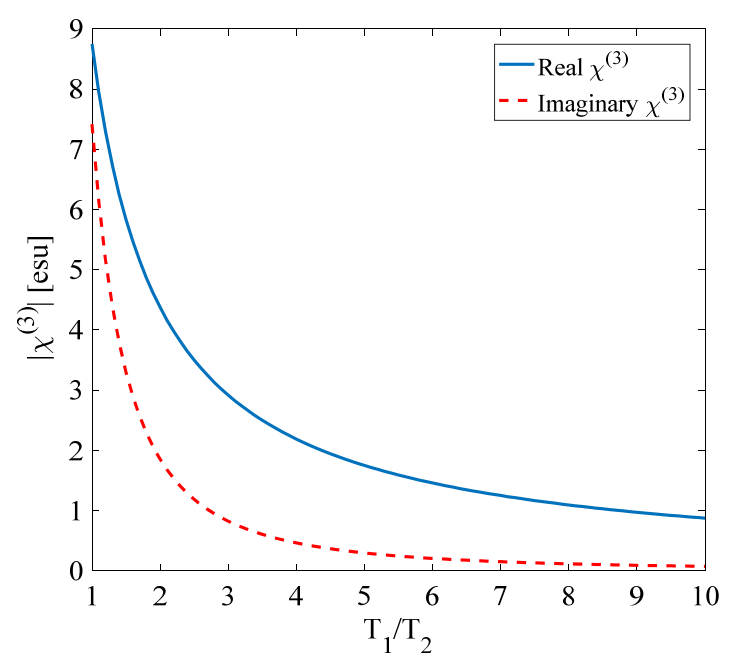

Figure 6. Third-order nonlinear optical susceptibility vs. population loss factor $T_{1} / T_{2}$ in the laser annealed $\mathrm{ZnO}$ nanostructures.

The encrypted signals correspond to the nonlinear optical parameters related to the fingerprints of the sample studied. The encryption method is proposed to be made by a systematic modification in particular regions of the film sample. The irradiation of a single pulse in different regions of the sample was conducted in single-shot mode. Pulsed irradiation close to the ablation threshold in the $\mathrm{ZnO}$ nanostructures gives origin to a strong change in the optical nonlinearities of the $\mathrm{ZnO}$ material. However, microscopy or spectroscopy studies are not able to clearly identify this modification in the sample.

The decryption stage was undertaken by the evaluation of the nonlinear optical absorption and optical Kerr effect in the nanostructures. XOR logic enables both encryption and decryption routes. Remarkably, by XOR encryption, the ciphering of the data can be guaranteed to be unbreakable without the clear identification of the two arguments involved in the logic gating.

Comparative results in $\mathrm{ZnO}$ nanostructures clearly pointed out their third-order nonlinear optical effects to be good candidates for building up high-throughput nanoscale platforms with potential applications in signal processing [29]. Collective interactions in $\mathrm{ZnO}$ nanostructures seem to be attractive for designing nonlinear optical nanosystems based on sculptured thin solid films [30]. We believe that our strategy opens up a perspective for flexible developing of all-optical functions with simultaneous actions related to absorptive and refractive nonlinearities. In particular, laser irradiation processes in $\mathrm{ZnO}$ nanostructures can be considered for improving manufacturing methods related to the implementation of dynamic multilevel diffractive optical elements. It can be envisioned that the coupling between nanostructures and nonlinearities could lead to exciting venues that may play an essential role in all-optical devices.

\section{Conclusions}

Polarization-selectable nonlinear optical absorption and nonlinear refraction were analyzed for developing a multipath load balancing technique by nanosecond pulses in a pump-probe configuration. The inherent modification of a third-order nonlinear optical response exhibited by optically irradiated $\mathrm{ZnO}$ nanostructures was studied by a detuning approximation. A two-wave mixing allowed us to identify two-photon absorption processes together with the optical Kerr effect as the third-order optical nonlinearities exhibited in different regions of the $\mathrm{ZnO}$ films. The systematic reading and recording of data as the real and imaginary part of the third-order nonlinear optical in the samples was obtained by nanosecond pulses featuring logic gate functions. The impact of nonlinear optical phenomena in low-dimensional materials seems to be suitable for designing platforms and dynamic tools for sensing and processing multiscale signals. 
Author Contributions: F.O.-T. carried out nonlinear optical experiments; M.T.-V. is responsible for the preparation and structure characterization of the samples; J.P.C.-L. analyzed photodamage effects; J.H.C.-C. analyzed nonlinear optical functions; C.T.-T. elucidated the application of the nonlinear optical properties and designed the paper. The manuscript was written through contribution of all authors. All authors have read and agreed to the published version of the manuscript.

Funding: This research was funded by Consejo Nacional de Ciencia y Tecnología (CONACyT) (grant No. CB-2015-251201), Comisión de Operación y Fomento de Actividades Académicas, Instituto Politécnico (COFAA) (2020) and Instituto Politécnico Nacional (IPN) (SIP-2020).

Acknowledgments: The authors kindly acknowledge the financial support from the Instituto Politécnico Nacional, COFAA-IPN, Universidad Nacional Autónoma de México, Universidad Politécnica del Valle de México and Consejo Nacional de Ciencia y Tecnología. The authors are also thankful to the Central Microscopy facilities of the Centro de Nanociencias y Micro y Nanotecnología del Instituto Politécnico Nacional.

Conflicts of Interest: The authors confirm that there are no conflicts of interest related to this research.

Data Availability: The authors declare that the data can be obtained upon request by emailing the corresponding author.

\section{References}

1. Kim, H.; Jung, K.; Yeo, S.J.; Chang, W.; Kim, J.J.; Lee, K.; Kim, Y.D.; Han, I.K.; Kwon, S.J. Long-distance transmission of broadband near-infrared light guided by a semi-disordered 2D array of metal nanoparticles. Nanoscale 2018, 10, 21275-21283. [CrossRef] [PubMed]

2. Cesca, T.; Calvelli, P.; Battaglin, G.; Mazzoldi, P.; Mattei, G. Local-field enhancement effect on the nonlinear optical response of gold-silver nanoplanets. Opt. Express 2012, 20, 4537-4547. [CrossRef]

3. Stepanov, A.L. Non-linear optical properties of metal nanoparticles implanted in silicate glass. Nucl. Instrum. Methods Phys. Res. Sect. B 2003, 206, 624-628. [CrossRef]

4. Chen, Y.; Lai, Z.; Zhang, X.; Fan, Z.; He, Q.; Tan, C.; Zhang, H. Phase engineering of nanomaterials. Nat. Rev. Chem. 2020. [CrossRef]

5. Kumar, S.S.; Venkateswarlu, P.; Rao, V.R.; Rao, G.N. Synthesis, characterization and optical properties of zinc oxide nanoparticles. Int. Nano Lett. 2013, 3, 1-6. [CrossRef]

6. Chen, J.; Qiu, Y.; Yang, D.; She, J.; Wang, Z. Improved piezoelectric performance of two-dimensional ZnO nanodisks-based flexible nanogengerators via ZnO/Spiro-MeOTAD PN junction. J. Mater. Sci. Mater. Electron. 2020, 31, 5584-5590. [CrossRef]

7. Fanizzi, F.P.; Jiang, J.; Pi, J.; Cai, J. The Advancing of Zinc Oxide Nanoparticles for Biomedical Applications. Bioinorg. Chem. Appl. 2018, 2018, 1062562. [CrossRef]

8. Kaushik, V.; Rajput, S.; Kumar, M. Broadband optical modulation in a zinc-oxide-based heterojunction via optical lifting. Opt. Lett. 2020, 45, 363-366. [CrossRef]

9. Serrà, A.; Gómez, E.; Philippe, L. Bioinspired ZnO-Based Solar Photocatalysts for the Efficient Decontamination of Persistent Organic Pollutants and Hexavalent Chromium in Wastewater. Catalysts 2019, 9, 974. [CrossRef]

10. Zhong, J.; Vogelsang, J.; Yi, J.; Wang, D.; Wittenbecher, L.; Mikaelsson, S.; Korte, A.; Chimeh, A.C.; Arnold, L.; Schaaf, P.; et al. Nonlinear plasmon-exciton coupling enhances sum-frequency generation from a hybrid metal/semiconductor nanostructure. Nat. Commun. 2020, 11, 1464. [CrossRef]

11. Khokhra, R.; Bharti, B.; Lee, H.; Kumar, R. Visible and UV photo-detection in ZnO nanostructured thin films via simple tuning of solution method. Sci. Rep. 2017, 7, 15032. [CrossRef] [PubMed]

12. Kelly, K.L.; Coronado, E.; Zhao, L.L.; Schatz, G.C. The optical properties of metal nanoparticles: The influence of size, shape, and dielectric environment. J. Phys. Chem. B 2003, 107, 668-677. [CrossRef]

13. Llopis-Lorente, A.; Díez, P.; Sánchez, A.M.; Marcos, D.; Sancenón, F.; Martínez-Ruiz, P.; Villalonga, R.; Martínez-Máñez, R. Interactive models of communication at the nanoscale using nanoparticles that talk to one another. Nat. Commun. 2017, 8, 15511. [CrossRef]

14. Can-uc, B.; Rangel-rojo, R.; Rodríguez-Fernández, L.; Oliver, A. Polarization selectable nonlinearities in elongated silver nanoparticles embedded in silica. Opt. Mater. Express 2013, 3, 2012-2021. [CrossRef]

15. di Ventra, M.; Stephane, E.; Heflin, J. Introduction to Nanoscale Science and Technology; Springer: Berlin/Heidelberg, Germany, 2004. 
16. Can-Uc, B.; López, J.; Lizarraga-Medina, E.G.; Borbon-Nuñez, H.A.; Rangel-Rojo, R.; Marquez, H.; Tiznado, H.; Jurado-González, J.A.; Hirata-Flores, G. Third-order nonlinear optical properties of a multi-layer Al2O3/ZnO for nonlinear optical waveguides. Opt. Express 2019, 27, 17359-17368. [CrossRef]

17. Rasool, A.; Kumar, M.C.S.; Mamat, M.H.; Gopalakrishnan, C.; Amiruddin, R. Analysis on different detection mechanisms involved in ZnO-based photodetector and photodiodes. J. Mater. Sci. Mater. Electron. 2020, 31, 7100-7113. [CrossRef]

18. Wang, K.; Zhou, J.; Yuan, L.; Tao, Y.; Chen, J.; Lu, P.; Wang, Z.L. Anisotropic Third-Order Optical Nonlinearity of a single ZnO Micro/Nanowire. Nano Lett. 2012, 12, 833-838. [CrossRef]

19. Gupta, J.; Bhargava, P.; Bahadur, D. Fluorescent $\mathrm{ZnO}$ for imaging and induction of DNA fragmentation and ROS-mediated apoptosis in cancer cells. J. Mater. Chem. B 2015, 3, 1968-1978. [CrossRef]

20. Zhang, L.; Wang, Y.; Wu, H.; Hou, M.; Wang, J.; Zhang, L.; Liao, C.; Liu, S.; Wang, Y. A ZnO nanowire-based microfiber coupler for all-optical photodetection applications. Nanoscale 2019, 11, 8319-8326. [CrossRef]

21. Castro-Chacón, J.H.; Torres-Torres, C.; Khomenko, A.V.; García-Zárate, M.A.; Trejo-Valdez, M.; Martínez-Gutiérrez, H.; Torres-Martínez, R. Encryption of nonlinear optical signals in ZnO:Al thin films by ultrashort laser pulses. J. Mod. Opt. 2017, 64, 601-608. [CrossRef]

22. Yatsui, T.; Jeong, H.; Ohtsu, M. Controlling the energy transfer between near-field optically coupled $\mathrm{ZnO}$ quantum dots. Appl. Phys. B 2008, 93, 199-202. [CrossRef]

23. Torres-Torres, C.; Castañeda, L.; Trejo-Valdez, M.; Maldonado, A.; Torres-Martínez, R. Participation of the Third Order Optical Nonlinearities in Nanostructured Silver Doped Zinc Oxide Thin Solid Films. J. Nanomater. 2012, 2012, 353061. [CrossRef]

24. Boyd, R.W. Nonlinear Optics, 3rd ed.; Academic Press: San Diego, CA, USA, 2008.

25. Sheik-Bahae, M.; Said, A.A.; Wei, T.; Hagan, D.J.; Stryland, E.W.V. Sensitive measurement of optical nonlinearities using a single beam. IEEE J. Quantum Electron. 1990, 26, 760-769. [CrossRef]

26. Chen, L.; Cui, Y.; Xiong, Z.; Zhou, M.; Gao, Y. Chemical functionalization of the ZnO monolayer: Structural and electronic properties. RSC Adv. 2019, 9, 21831-21843. [CrossRef]

27. Yannopapas, V. Enhancement of nonlinear susceptibilities near plasmonic metamaterials. Opt. Commun. 2010, 283, 1647-1649. [CrossRef]

28. Trejo-Valdez, M.; Torres-Torres, C.; Castro-Chacón, J.H.; Graciano-Armenta, G.A.; García-Gil, C.I.; Khomenko, A.V. Modification of the picosecond optical absorptive nonlinearity by a nanosecond irradiation in a nanostructured $\mathrm{ZnO}$ thin film. Opt. Laser Technol. 2013, 49, 75-80. [CrossRef]

29. Multian, V.V.; Riporto, J.; Urbain, M.; Mugnier, Y.; Djanta, G.; Beauquis, S.; Galez, C.; Gayvoronsky, Y.V.; le Dantec, R. Averaged third-order susceptibility of $\mathrm{ZnO}$ nanocrystals from Third Harmonic Generation and Third Harmonic Scattering. Opt. Mater. 2018, 84, 579-585. [CrossRef]

30. Karvonen, L.; Säynätjoki, A.; Chen, Y.; Jussila, H.; Rönn, J.; Ruoho, M.; Alasaarela, T.; Kujala, S.; Norwood, R.A.; Peyghambarian, N.; et al. Enhancement of the third-order optical nonlinearity in $\mathrm{ZnO} / \mathrm{Al}_{2} \mathrm{O}_{3}$ nanolaminates fabricated by atomic layer deposition. Appl. Phys. Lett. 2013, 103. [CrossRef]

(C) 2020 by the authors. Licensee MDPI, Basel, Switzerland. This article is an open access article distributed under the terms and conditions of the Creative Commons Attribution (CC BY) license (http://creativecommons.org/licenses/by/4.0/). 